\title{
INVESTIGACIONES
}

\section{Creencias de directores de establecimientos educacionales municipales acerca del compromiso docente}

\author{
Beliefs of municipal school principals about teacher commitment \\ Fresia Pino-Arcos ${ }^{a}$, Diana Pasmanik ${ }^{b}$ \\ ${ }^{a}$ Docente adscrita al Área de Psicología Educacional de la Escuela de Psicología \\ de la Universidad de Santiago de Chile. fresia.pino@usach.cl \\ ${ }^{b}$ Escuela de Psicología, Universidad de Santiago de Chile.diana.pasmanik@usach.cl
}

\begin{abstract}
RESUMEN
Este estudio se inscribe en la investigación orientada a avanzar en la comprensión de las relaciones entre el profesorado y el liderazgo directivo como elementos vinculados a la mejora educativa, enfocándose específicamente en el fenómeno del compromiso docente. Indaga, desde un enfoque cualitativo, en las creencias de directores de establecimientos educacionales, respecto de dicho compromiso. Los resultados indican que el compromiso será entendido como un atributo profesional, una forma de valoración del rol docente, que se expresa como una entrega extraordinaria hacia los estudiantes, el aprendizaje y la escuela. Emergen, además, creencias sobre la docencia, los docentes y el sistema municipal como componentes de un sistema de creencias. Las creencias muestran un compromiso docente idealizado, contrastando con las creencias acerca de los docentes, caracterizadas por un fuerte acento negativo. Se genera así una discrepancia con posibles consecuencias para las prácticas de liderazgo que justifican una mayor indagación.
\end{abstract}

Palabras claves: directores, creencias, compromiso docente, docentes, escuelas municipales.

\begin{abstract}
This study is part of research targeted to the advancement in the understanding of teachers and principals' leadership relationship as elements linked to education improvement, focused specifically on teachers' commitment. It enquires, by means of a qualitative approach, school principals' beliefs concerning this commitment. Results show that commitment is understood as a professional attribute, a way of valuing teaching's role, expressed as an extraordinary devotion to students, learning and school. There also emerge beliefs about teaching, teachers and the municipal system as components of a beliefs system. Beliefs show an idealized teachers' commitment, contrasting with beliefs concerning teachers, characterized by a strong negative accent. Therefore, a discrepancy is generated with eventual consequences for leadership practices that justify farther investigation.
\end{abstract}

Keywords: school principals, beliefs, teacher commitment, teachers, municipal schools. 
Estudios Pedagógicos XLVII N 1: 71-90, 2021

CREENCIAS DE DIRECTORES DE ESTABLECIMIENTOS EDUCACIONALES MUNICIPALES ACERCA DEL COMPROMISO DOCENTE

\section{INTRODUCCIÓN}

En el contexto de la investigación educativa en Chile, se ha demostrado que a través de la implementación de reformas educativas y específicamente en el nivel de instituciones educativas, los factores "profesor" y "liderazgo directivo" son las variables que mayor peso tienen sobre los resultados de los estudiantes (Tapia, Becerra, Mancilla \& Saavedra, 2011; Weinstein, 2009). Recientemente un estudio de Horn y Murillo (2016), basado en una amplia muestra de escuelas chilenas, declara que la influencia del liderazgo en los resultados de aprendizaje es viabilizada por el compromiso docente. Quiere decir que las prácticas de liderazgo influyen en el compromiso de los docentes, y se cuenta a su vez con evidencia empírica para señalar que este compromiso tiene influencia en el aprendizaje de los estudiantes (Murillo, Martínez \& Hernández, 2011; Altun, 2017).

Así lo señalan también revisiones de literatura internacional en las últimas dos décadas (Sun, 2014), que ubican el compromiso docente como un punto de conexión entre el liderazgo escolar y los resultados de aprendizaje, indicando su valor único ya que contribuye significativamente al aprendizaje de los estudiantes, mientras es maleable por el liderazgo del director. La influencia de esto último también tiene respaldo en la literatura especializada (Cansoy \& Polatcan, 2019; Cherkowski, 2012; Liu, 2015; Shila \& Sevilla, 2015).

En este sentido, resulta relevante aproximarse al fenómeno del compromiso docente desde una mirada que involucre a los agentes del contexto, teniendo a la vista que, al igual que otros elementos que conforman la docencia, el compromiso se expresa en contextos y realidades concretas (culturas y centros escolares) y adquiere sentido desde las condiciones que dichos contextos ofrecen, a partir de la implementación de políticas educativas en los sistemas educacionales. Este trabajo se enfoca en directores de escuela como agentes de relevancia reconocida, específicamente en sus creencias acerca del compromiso docente.

\subsection{COMPROMISO DOCENTE}

Firestone y Pennell (1993) definieron el compromiso como un estado voluntario en que la motivación intrínseca hacia los objetivos y valores de una institución (escuela) inspira esfuerzos más allá de las expectativas mínimas. Con ello se releva un enfoque más bien relacional, que observa el compromiso docente según el contexto en el que se expresa, teniendo en cuenta que estos contextos siempre estarán vinculados al acto educativo.

Datos y sistematizaciones posteriores acerca del compromiso docente lo describen como una dimensión personal que está mediada por otras características personales, pero que interactúa con variables presentes en el trabajo docente, incluyendo la identidad (personal y profesional), la autoeficacia, la motivación y la satisfacción con el trabajo (Crosswell, 2006). Aproximaciones más recientes en la investigación han identificado diferentes dimensiones para definirlo (institucional, personal y profesional), situándolo como un poderoso articulador de la identidad profesional (Fuentealba \& Imbarack, 2014).

La revisión de la literatura sobre compromiso docente da cuenta de clasificaciones sobre los objetos de compromiso, es decir, aquellos elementos hacia los cuales los docentes declaran sentirse comprometidos. Al respecto, existiría un acuerdo general en que dichos elementos son frecuentemente externos al docente: la escuela o la organización, los estudiantes, la continuidad de la carrera (entendida como la posibilidad de "hacer carrera" en la docencia), el conocimiento profesional de base, la profesión docente (Crosswell \& 
Elliot, 2004). Revisiones más actuales que sintetizan los significados del compromiso usados en los estudios empíricos (Sun, 2014; Hussen, Awgichew \& Thesome, 2016) identifican cuatro grandes dimensiones: Compromiso con la enseñanza, compromiso con los estudiantes, compromiso con la escuela y compromiso con el cambio. Hallazgos recientes han mostrado la relación entre compromiso docente no solo con el aprendizaje de los estudiantes, sino también con su disciplina (Mwaniki, Kiumi \& Ngunjiri, 2018). En términos más globales, el compromiso de los maestros con la enseñanza, los estudiantes y la escuela, contribuiría positivamente al aprendizaje exitoso, la calidad de la instrucción de los maestros, el crecimiento moral de los estudiantes y los logros académicos (Sun \& Leithwood, 2015).

Sin embargo, también se han desarrollado líneas de investigación que sugieren la existencia de una relación crucial entre al apego emocional al trabajo de enseñar y los niveles de compromiso personal de los docentes (Crosswell \& Elliot, 2004). Esta literatura constituye un reto a la visión que sitúa al compromiso docente como exclusivamente relacionado con dimensiones externas y pone en la línea de discusión la relación entre pasión, valores y creencias, y el compromiso docente. Se trata entonces de repensarlo más allá de los factores que lo determinan, e involucrar en su comprensión aquellos aspectos que obedecen a configuraciones identitarias que se construyen y reconstruyen a lo largo del tiempo.

\subsection{LIDERAZGO EDUCATIVO Y COMPROMISO DOCENTE}

En el contexto de las prácticas educativas, el compromiso docente no puede entenderse como un elemento aislado, que depende exclusivamente del propio docente. Es necesario incorporar una mirada contextual que visibilice las redes al interior de cada comunidad en el que este compromiso se expresa y por medio de las cuales va adquiriendo sentido como potenciador o mediador del cambio educativo, su valor como variable crítica en los procesos de mejora. Esto remite a su relación con el liderazgo educativo, teniendo a la vista que la acción y mirada de los directivos escolares van configurando modos de administrar la organización de las escuelas, que podrían o no favorecer mayores espacios de protagonismo y responsabilidad de parte de los docentes, movilizándolos hacia una construcción compartida de metas y objetivos educativos.

La evidencia empírica aportada por la investigación en el contexto internacional ha señalado una fuerte relación entre el liderazgo del director y el compromiso docente. Básicamente, se ha demostrado que los directores influyen en el compromiso de los docentes hacia sus escuelas (Cansoy \& Polatcan, 2019; Sun, 2014), y que los apoyos de los directores son fuente de compromiso profesional (Shoaib \& Khalid, 2017). Goddard, Goddard, Kim y Miller (citado en Bolívar, 2015) encontraron que había una relación directa significativa entre el liderazgo educativo de los directores y el grado de colaboración y trabajo conjunto de los docentes, y en este sentido el compromiso sería nutrido desde la conformación de una comunidad profesional de aprendizaje, a través del fortalecimiento de las creencias en la eficacia colectiva (Bolívar, 2015; Sun, 2014). Esto además es relevante, al considerar que aspectos como la confianza y cohesión, en el contexto de una comunidad profesional, han demostrado ser cruciales para mejorar los resultados en escuelas de bajo rendimiento (Shirrel, 2016). En sentido inverso, Doğan y Çelik (2019) encontraron que la razón por la que el compromiso organizacional de los docentes es bajo, proviene de una comunicación 
insuficiente con los directores de escuela, sus problemas en relaciones humanas, su falta de visión y el permanecer cerrados al desarrollo personal y organizacional.

De modo más específico, existe evidencia que vincula el estilo de liderazgo, específicamente el liderazgo transformacional, con el compromiso afectivo hacia la organización y la motivación autónoma de los maestros (Berkovich \& Eyal, 2017). Así mismo, en la medida en que los docentes sienten que sus directores se preocupan, los involucran, los apoyan y desarrollan, se incrementa su compromiso con la organización (Shila \& Sevilla, 2015).

La revisión de las investigaciones señala, además, que la percepción de un maestro del liderazgo del director influye en su compromiso, al igual que el modo en que los maestros entienden el sistema de valores del director y cómo este sistema se ajusta con sus propias orientaciones valóricas (Sun, 2014). El mismo autor propone, en términos más generales, que el compromiso docente se constituye como una especie de "ruta", a través de la cual la influencia de los directores "viaja", para expresarse en los resultados de aprendizaje de los estudiantes. Por lo tanto, resultaría relevante abrir preguntas o profundizar acerca de cómo este camino se produce.

En Chile, la investigación sobre liderazgo educativo es relativamente reciente, aunque ha cobrado en los últimos años mayor importancia situándose a la cabeza en el contexto latinoamericano (Horn \& Murillo, 2016). Existe evidencia en nuestro medio sobre la importancia del liderazgo en las motivaciones docentes (Valenzuela \& Horn, 2012; Reeves, 2012), pero aún no se cuenta con suficiente investigación que aborde la relación entre los líderes escolares y el compromiso docente. Tampoco respecto de la percepción de los directores ni de los factores de contexto de la escuela que podrían estar presentes en dicha relación. Recientemente, la investigación de Horn y Murillo (2016) buscó identificar en escuelas chilenas qué practicas del liderazgo directivo mostraban una mayor incidencia en el compromiso, llegando a establecer, según sus datos, que se trata de acciones que estructuran la escuela (preocuparse por establecer la misión y visión de la escuela), y acciones de apoyo pedagógico (jefes técnicos que se ocupan de que los docentes desarrollen un mejor trabajo).

\subsection{CREENCIAS: VALOR DE SU ESTUDIO PARA LA INVESTIGACIÓN EDUCATIVA}

La comprensión de las prácticas educativas desde los sistemas de creencias, representaciones, concepciones, o teorías implícitas, se adscribe al paradigma del pensamiento del profesor. Con él, se da lugar a un tipo de conocimiento que es más fiel a la complejidad, singularidad, incertidumbre y conflicto de valores que caracterizan la práctica profesional de la enseñanza (Jiménez \& Feliciano, 2006).

El concepto de creencia como constructo del ámbito de pensamiento remite a Dewey, quien en 1933 señala que una creencia:

Abarca todas las cuestiones acerca de las cuales no disponemos de un conocimiento seguro, pero en las que confiamos lo suficiente como para actuar de acuerdo con ellas, y también cuestiones que ahora aceptamos como indudablemente verdaderas, como conocimiento, pero que pueden ser cuestionadas en el futuro, de la misma manera que ocurrió con lo que en el pasado se tenía por conocimiento y hoy ha quedado relegado al limbo de la mera opinión o del error (Dewey, 1989, p. 24). 
Pajares (1992), en una sistematización -hoy en día clásica- con respecto a las creencias del profesorado en la investigación educacional, las describe como un constructo confuso ("messy"), y se refiere a ellas como conocimientos subjetivos, poco elaborados, que se generan a nivel individual para explicarse y justificar muchas de las decisiones y actuaciones, tanto personales como profesionales. No se fundamentan en la racionalidad sino más bien en los sentimientos, las experiencias y la ausencia de conocimientos específicos relativos al tema con que se relacionan. De este modo resultan ser consistentes y persisten en el tiempo.

De modo más específico, se ha hablado de creencias pedagógicas, definiéndolas como un conjunto de esquemas potentes sobre la educación, el conocimiento, el aprendizaje y la enseñanza a partir de las cuales los docentes toman decisiones conscientes y priorizadas (Fickel, 1999), teniendo incluso un mayor peso que los conocimientos disciplinarios que ellos poseen (Díaz, Martínez, Roa \& Sanhueza, 2010).

En cuanto a su organización, las creencias se van conectando y relacionando con otras creencias u otras estructuras cognitivas o afectivas (Rojas, 2014), y a su vez en una compleja red de construcciones intersubjetivas en que los sujetos participan, generando una comprensión estable de los procesos educativos que les corresponde abordar.

Son numerosas las investigaciones que avalan la idea de que las creencias afectan de modo determinante la conducta de los individuos y son los mejores indicadores de las decisiones que las personas realizan a lo largo de sus vidas (García \& Sebastián, 2011). En este último aspecto radicaría el valor de su estudio, en cómo inciden en las acciones de los profesionales de la educación.

La investigación reciente en la materia ha permitido atribuir a las creencias la capacidad de influir en la práctica pedagógica (Arnett \& Mady, 2018; Hutner \& Markman, 2016) y, al mismo tiempo, ser influida por ésta (Hutner \& Markman, 2016). Esto último suma, a la investigación mediante entrevistas y encuestas, aquella que incorpora la observación de la praxis pedagógica.

En este sentido, se puede comprender el potencial de cambio de las creencias en los escenarios de reflexión profesional, visualizando y explicitando una relación circular entre creencias que sustentan la acción y acciones que reestructuran creencias (Mellado \& Chaucono, 2015). La intención de hacer explícito lo que tradicionalmente se ha mantenido implícito, es lo que ha guiado el estudio de creencias en el contexto educativo, con miras a desarrollar estrategias que propicien cambios duraderos y de fondo en la educación (Aguilar, 2003).

Cabe señalar que en Chile, aunque se han venido desarrollando estudios sobre creencias en el profesorado y en estudiantes de pedagogía, las creencias de quienes ocupan cargos directivos han estado ausentes de la investigación sobre el tema (Mellado \& Chaucono, 2015).

El propósito de esta investigación es aportar elementos que permitan profundizar en lo que los líderes escolares piensan sobre el compromiso de los profesores, relevando desde un enfoque cualitativo, la voz de los sujetos. Esto implica reconocer que las formas que adquiere el ejercicio del liderazgo desde los sujetos en contexto, están ancladas en sus visiones de la escuela y de la docencia, así como en su experiencia con los docentes. El objetivo, por tanto, es describir las creencias relativas al compromiso docente que se manifiestan en el discurso de los directores de escuela.

En específico, a través de la investigación sobre las creencias de los directores de escuela relativas al compromiso docente, se esperaría contribuir a la comprensión del 
fenómeno a la vez que se reconoce el valor del pensamiento de los directores que, desde la gestión, se implican en sus comunidades escolares.

\section{METODOLOGÍA ${ }^{1}$}

Este estudio tiene por finalidad avanzar en la comprensión de un fenómeno, el compromiso docente, aportando con una indagación en las creencias de un grupo de directores de establecimientos educacionales municipales de enseñanza básica y media de la Región Metropolitana de Santiago de Chile, que atienden a poblaciones escolares con importantes niveles de vulnerabilidad escolar (IVE). Su diseño es descriptivo-interpretativo, el que se considera una estrategia investigativa pertinente para estudios orientados a responder preguntas de carácter exploratorio y con ello describir algún aspecto del fenómeno del que se ocupan (Vasilachis, 2009).

La opción por enfocar este estudio en la educación municipal obedece a que la función directiva resulta más relevante donde las condiciones escolares son más complejas (Bolívar, 2009). En Chile, son estos establecimientos los que concentran en mayor proporción a la población de menores ingresos y de mayor vulnerabilidad social (Raczynski, 2012). Al mismo tiempo, el sistema municipal se caracteriza por un alto grado de centralización, tanto en la gestión académica, en su dependencia técnica del Ministerio de Educación, como por su subordinación económica a los sostenedores municipales (Garay \& Uribe, 2006). En ese sentido, el compromiso docente -y las creencias que se le relacionen- en el caso particular de directores de instituciones educativas municipales, posee como un componente de su marco contextual las características del sistema en el cual docentes y directivos se encuentran insertos.

\subsection{PARTICIPANTES}

Teniendo estos antecedentes en consideración, la selección de los participantes se realizó mediante un muestreo intencionado, enfocándose en directores y directoras de establecimientos educacionales de educación básica y/o media de la Región Metropolitana con índice de vulnerabilidad escolar (IVE) superior al 70\%, en relación a su matrícula, que presentaran una diversidad en edad, sexo y experiencia. De este modo, se escogieron seis participantes, cuyas características se resumen en la tabla 1, mientras que en la tabla 2 se caracteriza a los establecimientos educacionales que dirigen.

Este artículo se origina a partir de los datos recolectados en la tesis para la obtención del grado de magister de la autora principal (Pino, 2019); la coautora fue su profesora guía. 
Tabla 1. Descripción de los participantes

\begin{tabular}{|c|c|c|c|c|}
\hline PARTICIPANTE & PROFESION & $\begin{array}{c}\text { TIEMPO EN EL } \\
\text { CARGO ACTUAL }\end{array}$ & $\begin{array}{c}\text { EXPERIENCIA } \\
\text { COMO DOCENTE } \\
\text { DE AULA }\end{array}$ & $\begin{array}{c}\text { ESTUDIOS } \\
\text { POSTERIORES }\end{array}$ \\
\hline 1 & $\begin{array}{c}\text { Profesora de Educación } \\
\text { General Básica }\end{array}$ & 4 años & 30 años & Magister en Educación \\
\hline 2 & Profesor de Castellano & 8 años & 7 años & Magister en Gestión \\
\hline 3 & $\begin{array}{c}\text { Profesor de Educación } \\
\text { General Básica }\end{array}$ & 3 años & 6 años & Magister en Educación \\
\hline 4 & Profesor de Castellano & 4 años & 2 años & Magister en Educación \\
\hline 5 & $\begin{array}{c}\text { Profesor de Educación } \\
\text { General Básica }\end{array}$ & 4 años & 25 años & $\begin{array}{c}\text { Magister en Currículum } \\
\text { y Liderazgo Educativo }\end{array}$ \\
\hline 6 & $\begin{array}{c}\text { Profesora de Educación } \\
\text { General Básica }\end{array}$ & 17 años & 10 años & Magister en Gestión \\
\hline
\end{tabular}

Fuente: Elaboración propia.

Tabla 2. Caracterización de los establecimientos educacionales que dirigen

\begin{tabular}{|c|c|c|c|c|c|}
\hline PARTICIPANTE & $\begin{array}{c}\mathbf{N}^{\mathbf{0}} \text { DE DOCENTES } \\
\text { EN EL } \\
\text { ESTABLECIMIENTO }\end{array}$ & $\begin{array}{c}\text { NIVEL } \\
\text { EDUCATIVO QUE } \\
\text { IMPARTE }\end{array}$ & $\begin{array}{c}\text { MATRÍCULA } \\
\text { TOTAL (2016) }\end{array}$ & $\begin{array}{c}\text { IVE } \\
\text { PROMEDIO } \\
\text { (2016) }\end{array}$ & COMUNA \\
\hline 1 & 27 profesores & Básica & 302 & $75,8 \%$ & Cerrillos \\
\hline 2 & 29 profesores & Básica y Media & 392 & $76,3 \%$ & La Florida \\
\hline 3 & 19 profesores & Básica & 209 & $85,6 \%$ & Cerrillos \\
\hline 4 & 60 profesores & Básica y Media TP & 344 & $83,3 \%$ & Cerrillos \\
\hline 5 & 60 profesores & Básica y media TP & 564 & $78,9 \%$ & La Florida \\
\hline 6 & 38 profesores & Básica & 405 & $72,3 \%$ & Santiago \\
\hline
\end{tabular}

Fuente: Elaboración propia.

La recolección de la información se efectuó mediante entrevistas semi-estructuradas individuales, con base en un guion temático. Esta técnica permite a los entrevistados expresarse en su propio lenguaje, en un relato elaborado desde su perspectiva y experiencias y abre la posibilidad de que incorporen aspectos no previstos por el entrevistador (Flores, 2009).

\subsection{ANÁLISIS DE LAS ENTREVISTAS}

El análisis de las entrevistas se orientó a partir de los lineamientos de la Teoría Fundamentada (Strauss y Corbin, 2002), considerando su potencial aporte en la generación de categorías conceptuales. Si bien está compuesta por tres niveles de codificación -abierto, axial y selectivopara fines del objetivo de esta investigación resultaba suficiente limitarse a los dos primeros. Conforme lo indica la Teoría Fundamentada, aplicando el método de las comparaciones constantes, se procedió a realizar una codificación abierta del texto transcrito de cada entrevista, generándose códigos que después fueron agrupados en categorías generales. 
El análisis de las entrevistas requería considerar los criterios que permitirían distinguir que un contenido determinado correspondía efectivamente a una creencia. Según DiegoMantecón, Blanco, Chamoso y Cáceres (2019), existe un cierto acuerdo en psicología con respecto a la definición de creencias; se les reconoce el ser subjetivas (específicas de cada individuo), construidas socialmente, organizadas en estructuras y representadas mentalmente bajo la forma de oraciones. De esto se deriva que la entrevista sea un recurso válido para estudiar creencias, requiriendo de la labor interpretativa de quien las investiga. Para esto último, además de su organización en estructuras, se consideraron como criterios su incidencia en los procesos de toma de decisiones (Pajares, 1992) y su diferenciación del conocimiento formal (o científico) al aludir a verdades personales, ligadas a evaluaciones o afectos sobre un determinado fenómeno y muy marcadas por la prescripción o el deber ser (Rojas, 2014).

De este modo, se buscó el contenido que refería al compromiso docente en sí mismo, en términos valorativos y prescriptivos, pero también a creencias que se relacionarían con éste, tales como las valoraciones relativas al profesorado en general y al cuerpo docente del establecimiento educacional. Estos mismos indicadores, aplicados a otros elementos contextuales y la argumentación para justificar la toma de decisiones, fueron también una fuente para identificar las creencias en el análisis de las entrevistas. De este modo, se efectuó el proceso de análisis del que emergieron categorías y subcategorías organizándose en ejes axiales.

\subsection{CRITERIOS DE RIGOR}

Como señalan Cornejo y Salas (2011), en un estudio cualitativo, su calidad científica se resguarda con la rigurosidad en su desarrollo, que incluye el grado de ajuste entre el objeto a investigar, el objetivo que guía la investigación y el enfoque investigativo, con sus métodos y técnicas. Guba (1989) propone cuatro criterios de rigor de la investigación cualitativa, que son los que guiaron esta investigación. Se resumen en la tabla 3.

Tabla 3. Criterios de rigor de la investigación cualitativa de Guba y Lincoln (1989)

\begin{tabular}{|c|c|c|}
\hline CRITERIOS & ENTENDIDOS COMO... & PROCEDIMIENTOS PROPUESTOS \\
\hline Credibilidad & $\begin{array}{c}\text { Cómo establecer confianza en la verdad de } \\
\text { los resultados }\end{array}$ & $\begin{array}{c}\text { Contrastación de las interpretaciones del } \\
\text { investigador con otras fuentes (participantes). }\end{array}$ \\
\hline Transferibilidad & $\begin{array}{c}\text { Formulación de hipótesis de trabajo que } \\
\text { puedan transferirse a otros contextos } \\
\text { similares }\end{array}$ & $\begin{array}{c}\text { Descripción detallada del contexto en que han } \\
\text { sido generado los resultados }\end{array}$ \\
\hline Dependencia & $\begin{array}{c}\text { Elementos de estabilidad de los datos y la } \\
\text { rastreabilidad de estos }\end{array}$ & $\begin{array}{c}\text { Descripción detallada del contexto en que han } \\
\text { sido generados los datos. }\end{array}$ \\
\hline Confirmabilidad & $\begin{array}{c}\text { Reducción del sesgo que producen las } \\
\text { motivaciones, intereses e inclinaciones del } \\
\text { investigador }\end{array}$ & $\begin{array}{c}\text { Triangulación. Verificación de los datos } \\
\text { producidos. }\end{array}$ \\
\hline
\end{tabular}

Fuente: Elaboración propia a partir de Guba (1989). 


\subsection{RESGUARDOS ÉTICOS}

Asimismo, para asegurar el desarrollo de un proceso de investigación ético, se contó con un consentimiento informado visado por el comité de ética institucional de la Vicerrectoría de Investigación, Desarrollo e Innovación de la Universidad de Santiago de Chile (Informe $\mathrm{N}^{\circ}$ 545). Además de su firma, se mantuvo una disposición ética en la investigación que permitiera a los entrevistados una participación libre e informada, y el tratamiento confidencial de los datos.

\section{RESULTADOS}

\subsection{DESCRIPCIÓN DE CATEGORÍAS}

A partir del análisis de las entrevistas, se identificaron dos ejes principales: creencias sobre el compromiso docente y elementos que contextualizan el compromiso docente. Se muestran los resultados organizados primariamente en estos ejes, y dentro de ellos en categorías y subcategorías que se derivaron de la codificación de los relatos (Tabla 4).

Tabla 4. Categorías y subcategorías

\begin{tabular}{|c|c|c|c|c|}
\hline \multicolumn{2}{|c|}{$\begin{array}{l}\text { A. CREENCIAS SOBRE EL } \\
\text { COMPROMISO DOCENTE }\end{array}$} & \multicolumn{3}{|c|}{$\begin{array}{l}\text { B. ELEMENTOS QUE CONTEXTUALIZAN EL } \\
\text { COMPROMISO DOCENTE }\end{array}$} \\
\hline $\begin{array}{l}\text { A1. Creencias sobre } \\
\text { qué es el compromiso } \\
\text { docente }\end{array}$ & $\begin{array}{l}\text { A2. Creencias sobre } \\
\text { cómo se manifiesta el } \\
\text { compromiso docente }\end{array}$ & $\begin{array}{l}\text { B1. Creencias sobre } \\
\text { los docentes }\end{array}$ & $\begin{array}{l}\text { B2. Creencias sobre } \\
\text { la docencia }\end{array}$ & $\begin{array}{l}\text { B3. Creencias sobre } \\
\text { el sistema municipal }\end{array}$ \\
\hline $\begin{array}{l}\text { Atributo personal - } \\
\text { intrínseco }\end{array}$ & $\begin{array}{l}\text { Relación con los } \\
\text { estudiantes }\end{array}$ & $\begin{array}{l}\text { Deficiente formación } \\
\text { profesional }\end{array}$ & Rol social & $\begin{array}{l}\text { Concentra al } \\
\text { profesorado con } \\
\text { menos vocación }\end{array}$ \\
\hline $\begin{array}{l}\text { Valoración del rol } \\
\text { profesional }\end{array}$ & $\begin{array}{l}\text { Cumplimiento apegado } \\
\text { al rol formal }\end{array}$ & Resistentes al cambio & $\begin{array}{l}\text { Trabajo que no tiene } \\
\text { gran impacto }\end{array}$ & $\begin{array}{l}\text { Población escolar en } \\
\text { condición de } \\
\text { vulnerabilidad }\end{array}$ \\
\hline Entrega & $\begin{array}{l}\text { Preocupación por el } \\
\text { aprendizaje }\end{array}$ & $\begin{array}{l}\text { No poner a los } \\
\text { estudiantes en primer } \\
\text { lugar }\end{array}$ & Vocación - Entrega & \\
\hline Foco en el aprendizaje & $\begin{array}{l}\text { Manejo de la disciplina } \\
\text { de aula }\end{array}$ & $\begin{array}{l}\text { Actitud de } \\
\text { confrontación con sus } \\
\text { contextos laborales }\end{array}$ & Liderazgo - Gestión & \\
\hline Con el estudiante & En la jefatura de curso & $\begin{array}{l}\text { Tendencia a } \\
\text { conducirse como } \\
\text { grupo }\end{array}$ & $\begin{array}{l}\text { Pérdida del respeto y } \\
\text { orgullo profesional }\end{array}$ & \\
\hline Con la escuela & $\begin{array}{l}\text { Adecuarse a las } \\
\text { condiciones }\end{array}$ & $\begin{array}{l}\text { Experimentan una } \\
\text { sensación de } \\
\text { injusticia }\end{array}$ & $\begin{array}{l}\text { Se nutre desde una } \\
\text { identidad histórica }\end{array}$ & \\
\hline Buscar mejorar & & $\begin{array}{l}\text { Desempeño } \\
\text { condicionado por la } \\
\text { modalidad } \\
\text { contractual }\end{array}$ & & \\
\hline \multicolumn{5}{|l|}{ Hacer la diferencia } \\
\hline Rasgo selectivo & & & & \\
\hline
\end{tabular}




\subsubsection{EJE A: Creencias sobre el compromiso docente}

En este eje se agruparon todos aquellos sentidos que, desde el relato de los entrevistados, pudieron desprenderse como parte de una concepción más global de la que serían parte y que contribuirían a formar sus creencias acerca del compromiso de los profesores. Por ejemplo, el cómo conceptualizan o definen este fenómeno, qué elementos les parecen esenciales o constitutivos, también el cómo se expresa en conductas y aspectos observables o interpretables del quehacer de los docentes.

A1: Creencias sobre qué es el compromiso docente: Se agrupan en esta categoría aquellas declaraciones con las que los entrevistados se refieren a aspectos que definen el compromiso docente.

Existe un amplio acuerdo entre los sujetos en definirlo como un atributo personal, algo que es intrínseco a cada docente, relacionado con la vocación y con los valores personales. En este sentido, se entiende que el compromiso docente sería algo personal antes que institucional.

También existe acuerdo en reconocer que está definido por la valoración del trabajo como profesor, la valoración del rol profesional y social que se desempeña, asociándolo a un quehacer con sentido, un quehacer con el cual hay que comprometerse a pesar de las condiciones en las que se ejerce la profesión, porque el compromiso estaría presente más allá de aspectos circunstanciales. Es entendido como una forma de otorgar valor al propio trabajo y al mismo tiempo desarrollar altas expectativas acerca del impacto que este trabajo tiene en los estudiantes.

Así, de un modo parcial, es decir, no compartido por todos los entrevistados, aparecen ideas relativas a: (a) la entrega, desde donde se asume que el compromiso es una forma de darse con los alumnos, estar dispuesto a todo, más allá de lo establecido por un contrato con el empleador; (b) el aprendizaje de los estudiantes, indicando que el compromiso sería la preocupación por el aprendizaje; (c) compromiso con los estudiantes en tanto sujetos de su labor, definiendo en este sentido el objeto del compromiso; (d) compromiso con la escuela, entendido como una forma de identificación con la institución; (e) el compromiso entendido como un "hacer la diferencia", generar cambios; (f) la búsqueda por mejorar profesionalmente, y en el mismo sentido, será definido como (e) un rasgo selectivo, algo que distingue a unos docentes de otros.

A manera de síntesis, puede señalarse que el compromiso docente es para los entrevistados un atributo que está anclado en el ser profesor, como una forma, en primer lugar, de valoración del rol que se desempeña, como una disposición hacia un algo (los estudiantes, la escuela, el aprendizaje), y que se vive como una entrega extraordinaria, más allá de lo que señalan los límites contractuales del trabajo remunerado. 
Tabla 5. Citas ilustrativas de las definiciones del compromiso docente

\begin{tabular}{|c|c|}
\hline $\begin{array}{l}\text { Atributo personal - } \\
\text { intrínseco }\end{array}$ & $\begin{array}{l}\text { "Si es una cosa personal, ya no es una cosa de tipo institucional, es el orgullo } \\
\text { profesional que pienso yo que debe tener toda persona". }(5,106) ; \text { "[...]que los } \\
\text { aprendizajes, [...] se construyen desde el corazón, que tiene que ver con un } \\
\text { compromiso real del profesor, del alma". }(2,66) ; \text { "[...] es como trabajar con } \\
\text { vocación". (1,62). }\end{array}$ \\
\hline $\begin{array}{l}\text { Valoración del rol } \\
\text { profesional }\end{array}$ & $\begin{array}{l}\text { "El sentido de compromiso del profesor, la valoración de su rol social, entendido } \\
\text { desde ese punto de vista, no solamente como una profesión que me permite ganarme } \\
\text { la plata". }(3,88) \text {. }\end{array}$ \\
\hline Entrega & $\begin{array}{l}\text { “[...] son dispuestos a todo, ahí tú te das cuenta que hay una entrega, más allá de lo } \\
\text { normal, más allá de lo que está estatuido. O sea, es un profesor que se queda, que le } \\
\text { ayuda a los niños en forma extraordinaria [...]”. (6,52) }\end{array}$ \\
\hline Foco en el aprendizaje & $\begin{array}{l}\text { "[...] yo creo que se define en que se preocupan de que sus alumnos aprendan, que } \\
\text { esa es la esencia de la educación". }(1,70)\end{array}$ \\
\hline Con el estudiante & $\begin{array}{l}\text { "[...] nosotros tenemos un compromiso con los estudiantes, y por eso nos van a } \\
\text { evaluar. [...] los estudiantes, por lo tanto, ese es nuestro principal... y lo demás, mira, } \\
\text { pasa a segundo lugar, tercer lugar, lo podemos conversar y todo, pero lo primordial } \\
\text { son los estudiantes". }(5,62)\end{array}$ \\
\hline Con la escuela & $\begin{array}{l}\text { “Acto del } 18 \text { de septiembre, se moviliza toda la escuela, tratando... más allá de los } \\
\text { horarios, más allá de todo". }(6,66)\end{array}$ \\
\hline Buscar mejorar & $\begin{array}{l}\text { "[...] una profesora que decide probarse a sí misma y avanzar un poco más, eso la } \\
\text { hace distinta". }(6,56)\end{array}$ \\
\hline Hacer la diferencia & $\begin{array}{l}\text { "[...] hay mucha gente que está comprometida, entonces hemos ido tratando de } \\
\text { cambiar algunas cosas". }(3,46)\end{array}$ \\
\hline Rasgo selectivo & $\begin{array}{l}\text { "[...] son profes súper constantes en todas las cosas, se nota inmediatamente que } \\
\text { tienen algo distinto, un sello }[\ldots] " .(1,64) \text {. }\end{array}$ \\
\hline
\end{tabular}

A.2. Creencias sobre cómo se manifiesta el compromiso docente: En esta categoría se reúnen las ideas de los entrevistados que darían cuenta de los modos en que el compromiso docente se expresa o se traduce en aspectos observables, o en acciones desde las cuales se puede inferir que el rasgo está presente en un profesor. Se destacan la relación que los docentes establecen con los estudiantes, una forma de trato que denota cercanía y calidez, y también como una forma de relación con el trabajo, que se expresaría en el cumplimiento apegado al rol formal que se asume.

Otros sentidos se refieren a observar el compromiso focalizado en una preocupación por el aprendizaje de los estudiantes, o en el trabajo que se realiza para manejar la disciplina de aula, creando un ambiente ordenado y acogedor. Esto viene a concretarse específicamente al señalar que el compromiso es más evidente cuando se asume el rol como profesor jefe de curso, mostrando un liderazgo que les permite hacerse cargo y gestionar aspectos más individuales de los estudiantes. Finalmente, aparece como una expresión del compromiso la capacidad del docente de adecuarse a las condiciones en que le toca enseñar, especialmente en contextos de vulnerabilidad social. 
Estudios Pedagógicos XLVII N 1: 71-90, 2021

CREENCIAS DE DIRECTORES DE ESTABLECIMIENTOS EDUCACIONALES MUNICIPALES ACERCA DEL COMPROMISO DOCENTE

Tabla 6. Citas ilustrativas de las manifestaciones del compromiso docente

\begin{tabular}{|l|l|}
\hline $\begin{array}{l}\text { Relación con los } \\
\text { estudiantes }\end{array}$ & $\begin{array}{l}\text { "Cuando los profesores se entregan de esa forma, mejora todo... mejoran ellos } \\
\text { profesionalmente, los estudiantes, que sienten que un profesor se la juega por ellos, que } \\
\text { es, como dicen ellos «Es aperrado, el profe, es seco»". (5,106). }\end{array}$ \\
\hline $\begin{array}{l}\text { Cumplimiento apegado } \\
\text { al rol formal }\end{array}$ & $\begin{array}{l}\text { "También el compromiso que se manifieste en que llegue, en que esté, en que cumpla y } \\
\text { en que haga las cosas bien". (4,76). }\end{array}$ \\
\hline $\begin{array}{l}\text { Preocupación por el } \\
\text { aprendizaje }\end{array}$ & "[...] que florezca en los aprendizajes, y en la relación con ellos". (2,140) \\
\hline $\begin{array}{l}\text { Manejo de la disciplina } \\
\text { de aula }\end{array}$ & $\begin{array}{l}\text { "[...] tú entras a la sala y se nota un aire diferente. Y los que son al revés, también se } \\
\text { nota inmediatamente, tú vas a esa sala y hay un aire diferente, los niños más } \\
\text { indisciplinados, las salas no acogedoras". (1,64). }\end{array}$ \\
\hline En la jefatura de curso & $\begin{array}{l}\text { "...] ellos tienen un liderazgo como profesores jefes y se hacen cargo de todo lo que } \\
\text { ocurre en el aula, y lo que ocurre fuera del aula". (5,68). }\end{array}$ \\
\hline $\begin{array}{l}\text { Adecuarse a las } \\
\text { condiciones }\end{array}$ & $\begin{array}{l}\text { "...] en estos colegios uno hace de todo [...]. Entonces la persona que contrate, } \\
\text { también tiene que estar dispuesta así [...] y si hay que sonarle los mocos a los cabros, } \\
\text { hay que sonarle los mocos a los cabros. O sea no, no se puede hacer otra cosa". (1,90). }\end{array}$ \\
\hline
\end{tabular}

\subsubsection{EJE B: Elementos que contextualizan el compromiso docente}

B1: Creencias sobre los docentes: En esta subcategoría se incorporaron las ideas que, desde su experiencia de trabajo, los directores se han ido formando sobre los docentes. Existiría un acuerdo general en que los profesores cuentan con una formación profesional deficiente, refiriéndose al poco dominio que tienen sobre las disciplinas que enseñan, algo que han observado directamente en sus prácticas, y al desconocimiento de los métodos adecuados para enseñar. También se cree no cuentan con suficientes herramientas para manejar a los grupos de estudiantes y, de un modo más global, se reconoce una formación deficiente en cuanto a la visión pedagógica, y al desconocimiento de la trascendencia, del rol social que tiene la labor docente. Se señala la resistencia de los docentes al cambio, lo que se expresa en la rigidez en sus prácticas o en el rechazo a los cambios en el entorno inmediato de su trabajo.

En cuanto a su trabajo, los entrevistados señalan que los docentes tienden a no poner a los alumnos en primer lugar, perdiéndolos de vista y descuidando a los alumnos como lo más importante de su labor. Más aún, se cree que los profesores anteponen sus intereses a las necesidades de los estudiantes. Este descuido se asociaría a la preocupación de los docentes por la reivindicación de sus derechos y también a los prejuicios que tienen sobre el origen socioeconómico de los estudiantes. También en este contexto, aparece la creencia sobre la actitud de confrontación de los docentes con sus contextos laborales, percibiendo que los docentes tienden a formular exigencias al sistema, a la vez que resienten los aspectos que les resultan demandantes. Se entiende, asimismo como una relación entre crítica y autocrítica, en la que suelen criticar o demandar soluciones en sus contextos mientras carecen de una mirada autocrítica respecto de su trabajo. Aquí aparece también una visión de los docentes en la que se cree que tienden a conducirse como grupo que influyen en las conductas individuales y que construyen percepciones compartidas que, por ejemplo, son plasmadas en las movilizaciones gremiales.

En relación a lo anterior, también se cree que los docentes experimentan una sensación de injusticia, según la cual, en el contexto de las complejidades y tensiones del ejercicio de la profesión, existiría una cultura de queja y suspicacia que surge de la 
sensación de recibir remuneraciones injustas, no sentirse reconocidos en su trabajo, y de ser injustamente responsabilizados por los pobres resultados que se conocen en educación. Algunos entrevistados declaran que el trabajo de sus profesores debe observarse a partir de estas complejidades, por ejemplo, considerar que es más difícil trabajar con estudiantes en situación de vulnerabilidad, y que en este sentido, el sistema les impone una exigencia para la que no estarían preparados.

Finalmente, se menciona que el trabajo de los docentes se muestra de forma distinta en función de las condiciones laborales, y que en este sentido su desempeño es condicionado por la modalidad contractual. De este modo, la seguridad o inseguridad que ofrece el tipo de contrato laboral se traduce en que, a juicio de algunos entrevistados, el desempeño de un profesor es mucho mejor cuando está bajo amenaza de perder el trabajo.

Tabla 7. Citas ilustrativas de las creencias sobre los docentes

\begin{tabular}{|c|c|}
\hline $\begin{array}{l}\text { Formación profesional } \\
\text { deficiente }\end{array}$ & $\begin{array}{l}\text { "Es deficiente, y es deficiente no solamente del punto de vista del manejo disciplinar, ya sea } \\
\text { de la asignatura que ejercen o del conocimiento que tengan sobre educación. Pienso que lo } \\
\text { que se ha perdido es la formación, esto que decíamos antes, de esta mística, de este sentido } \\
\text { de trascendencia del profesor, del rol social que yo juego... no es una cuestión que releven } \\
\text { las universidades". (3,86). }\end{array}$ \\
\hline Resistencia al cambio & $\begin{array}{l}\text { "[...] las estrategias pedagógicas, las prácticas, que sean más innovadores. Les cuesta } \\
\text { hacer cambios, les cuestan los cambios, las innovaciones pedagógicas". (1,118) }\end{array}$ \\
\hline $\begin{array}{l}\text { No poner a los } \\
\text { estudiantes en primer } \\
\text { lugar }\end{array}$ & $\begin{array}{l}\text { "Eso es abandono, o sea, yo me centro en mí, en mis deficiencias, y en lo injustos que han } \\
\text { sido con nosotros los profesores, y que los sueldos y que... y desecho. O sea, me centro en mí } \\
\text { y los cabros no aprenden". (2,80), "[... l la mirada que tiene un profe municipal, siempre es } \\
\text { pensando en ellos primero, después en los alumnos". }(2,12) \text {. }\end{array}$ \\
\hline $\begin{array}{l}\text { ctitud de } \\
\text { onfrontación con sus } \\
\text { ontextos laborales }\end{array}$ & $\begin{array}{l}\text { "Entonces el profesor es muy crítico, si hay algo que lo caracteriza, es que critica todo, todo } \\
{[\ldots] \text { Entonces no es un buen ejercicio, sustraerse, y enlodar la responsabilidad siempre en }} \\
\text { el otro, en el otro, en el otro. Entonces yo creo que eso los profesores en general, lo tenemos } \\
\text { poco desarrollado". (5,42). }\end{array}$ \\
\hline $\begin{array}{l}\text { Tendencia a conducirse } \\
\text { como grupo }\end{array}$ & $\begin{array}{l}\text { "Porque obviamente un director que te diga que no tiene grupos en un establecimiento, es } \\
\text { mentira. Hay grupos positivos y hay grupos negativos". }(6,58) \text {. }\end{array}$ \\
\hline $\begin{array}{l}\text { Experimentan } \\
\text { sensación de injusticia }\end{array}$ & $\begin{array}{l}\text { "[...] históricamente sienten que hay una deuda con el profesorado, por la situación de } \\
\text { remuneraciones de no ser reconocidos, no solamente como profesionales, sino que el respeto } \\
\text { que se ha perdido hacia ellos, se les ningunea, se les sindica como constantes responsables } \\
\text { directos de todo el deterioro que ha habido en educación". }(5,42)\end{array}$ \\
\hline $\begin{array}{l}\text { mpeño } \\
\text { cionado por la }\end{array}$ & $\begin{array}{l}\text { "[... ] el comportamiento del profesor en escuela particular subvencionada, como tiene esta } \\
\text { «espada de Damocles» en que tú sabes que si no rindes te vas, el resultado de su quehacer } \\
\text { es mucho mayor, aún en esas condiciones. Porque está... o sea, si no sirve se va”. (6,102). }\end{array}$ \\
\hline
\end{tabular}

B2. Creencias sobre la docencia: Se consideró esta subcategoría como uno de los elementos que proporcionan contexto al compromiso docente, dado que en ella se reúnen aspectos principalmente valorativos respecto a la docencia. Todas las visiones que emanaron de los relatos acerca de lo que la docencia es y debe ser, distinguiendo estos elementos de las creencias sobre los docentes en concreto, se consideraron creencias sobre la docencia. Su relevancia en relación al compromiso docente consistiría en que dichas creencias pueden estar operando como filtros para observar a los docentes como sujetos, observar su trabajo y su compromiso. De acuerdo a los elementos reunidos en este subgrupo, los sujetos exhiben 
creencias sobre (a) el rol social de la docencia, considerándola como una profesión destinada especialmente a provocar cambios en el ser humano, y desde ahí ocupar un lugar en el marco de las transformaciones sociales. Ser docente significaría ser un agente de cambio, y por lo mismo cada profesor debiese ser consciente de su rol social. Paradójicamente, existe también un alto acuerdo entre los entrevistados en considerar la docencia como (b) un trabajo que no tiene gran impacto en el entramado de las políticas educativas, y en comparación con la tarea de un director, es algo que conlleva menor responsabilidad.

Existen también sentidos que develan creencias sobre lo que la docencia es y sobre lo que debe ser un docente. Entendiendo la docencia entre otras cosas como: (c) una vocación y entrega, expresada como una forma de relación hacia los estudiantes como hacia el aprendizaje, y (d) como una forma de liderazgo, asociada a la capacidad de gestión de los cursos. También se señalan aspectos que han cambiado en relación a la docencia, como (e) la pérdida del respeto y orgullo profesional, así como (f) la creencia de que la docencia se nutre desde una identidad histórica que cobra sentido en la comunidad escolar en la que se trabaja.

Tabla 8. Citas ilustrativas de las creencias sobre la docencia

\begin{tabular}{|c|c|}
\hline & $\begin{array}{l}\text { "Es deficiente, y es deficiente no solamente del punto de vista del manejo disciplinar, ya sea } \\
\text { de la asignatura que ejercen o del conocimiento que tengan sobre educación. Pienso que lo } \\
\text { que se ha perdido es la formación, esto que decíamos antes, de esta mística, de este sentido } \\
\text { de trascendencia del profesor, del rol social que yo juego... no es una cuestión que releven las } \\
\text { universidades". (3,86). }\end{array}$ \\
\hline & $\begin{array}{l}\text { "[...] las estrategias pedagógicas, las prácticas, que sean más innovadores. Les cuesta hacer } \\
\text { cambios, les cuestan los cambios, las innovaciones pedagógicas”. }(1,118)\end{array}$ \\
\hline $\begin{array}{l}\text { No poner a los } \\
\text { estudiantes en primer } \\
\text { lugar }\end{array}$ & $\begin{array}{l}\text { "Eso es abandono, o sea, yo me centro en mí, en mis deficiencias, y en lo injustos que han sido } \\
\text { con nosotros los profesores, y que los sueldos y que... y desecho. O sea, me centro en mí y los } \\
\text { cabros no aprenden". (2,80), "[...] la mirada que tiene un profe municipal, siempre es } \\
\text { pensando en ellos primero, después en los alumnos". }(2,12) \text {. }\end{array}$ \\
\hline $\begin{array}{l}\text { ción con sus } \\
\text { laborales }\end{array}$ & $\begin{array}{l}\text { "Entonces el profesor es muy crítico, si hay alg } \\
{[\ldots] \text { Entonces no es un buen ejercicio, sustraer. }} \\
\text { otro, en el otro, en el otro. Entonces yo creo q } \\
\text { poco desarrollado". (5,42). }\end{array}$ \\
\hline & $\begin{array}{l}\text { "Porque obviamente un director que te diga que no tiene grupos en un establecimiento, es } \\
\text { mentira. Hay grupos positivos y hay grupos negativos". }(6,58) \text {. }\end{array}$ \\
\hline $\begin{array}{l}\text { Experimentan } \\
\text { sensación de injusticia }\end{array}$ & $\begin{array}{l}\text { "[...] históricamente sienten que hay una deuda con el profesorado, por la situación de } \\
\text { remuneraciones de no ser reconocidos, no solamente como profesionales, sino que el respeto } \\
\text { que se ha perdido hacia ellos, se les ningunea, se les sindica como constantes responsables } \\
\text { directos de todo el deterioro que ha habido en educación". (5,42) }\end{array}$ \\
\hline lodandaa contractue & $\begin{array}{l}\text { "[...] el comportamiento del profesor en escuela particular subvencionada, como tiene esta } \\
\text { «espada de Damocles» en que tú sabes que si no rindes te vas, el resultado de su quehacer es } \\
\text { mucho mayor, aún en esas condiciones. Porque está... o sea, si no sirve se va”. }(6,102) \text {. }\end{array}$ \\
\hline
\end{tabular}

B3. Creencias sobre el sistema municipal: Se consideró que el sistema municipal aportaba ciertos elementos asociados al quehacer de los docentes, que funcionaban también como contexto del compromiso. En este subgrupo se aprecian ideas que emergen de la experiencia de los sujetos al dirigir a sus equipos docentes específicamente en el contexto municipal, y cómo esta condición va a operar a la hora de valorar el trabajo de los docentes y sus 
características particulares. Surgen así creencias que pueden influir en qué es lo que se espera de los docentes y cuán favorable resulta este sistema en función de estimular el compromiso.

Un primer elemento lo constituye la creencia de que el sistema municipal concentra al profesorado con menor vocación, expresado en la frase "lo que bota la ola", y que los buenos profesores (con vocación) trabajan en establecimientos donde se les ofrece una mejor remuneración. El profesor con vocación sería un recurso escaso en el sistema municipal. También se caracteriza a los docentes del sistema municipal como tristes, quejosos, que piensan en ellos antes que en los estudiantes, que se centran en sus reclamos, y que por lo mismo muchas cosas deben negociarse con ellos. Por otra parte, se consideran significativas las condiciones sociales de la población escolar que atiende el sistema municipal. De este modo va a entenderse que el desempeñarse como profesor en un colegio municipal es una tarea más difícil que hacerlo en otro sistema, solamente por las características de sus estudiantes y las problemáticas psicosociales asociadas a la población escolar en condición de vulnerabilidad. Las condiciones de precariedad se extienden al establecimiento generando mayores dificultades, no solo por la escasez de recursos, sino por las trabas burocráticas que hacen más difícil acceder a mejoras materiales. Finalmente, se distinguen creencias que relacionan el contexto de educación municipal con la necesidad de atraer a buenos docentes, y consecuentemente ofrecer mejores remuneraciones, que compensen las dificultades que se experimentan al trabajar en este tipo de establecimientos.

Tabla 9. Citas ilustrativas de las creencias sobre sistema municipal

\begin{tabular}{|l|l|}
\hline Concentra al profesorado & $\begin{array}{l}\text { "Lo que vi no me gustó en cuanto a los docentes, o sea el docente como triste, docente } \\
\text { con la queja eterna". (1,10) } \\
\text { "Entonces aquí llega... en eso sí te podría decir yo, que llega lo que bota la ola a los } \\
\text { colegios públicos... los profesores buenos se van a otros lados, donde les pagan mejor, } \\
\text { obviamente. Porque los de vocación, no se vienen pa' acá, o sea, se podrían venir pero } \\
\text { son escasísimos, muy preciado recurso." }(2,20)\end{array}$ \\
\hline $\begin{array}{l}\text { Población escolar en } \\
\text { condición de vulnerabilidad }\end{array}$ & $\begin{array}{l}\text { "Es que la pega del profesor es una pega dura, es atender, como en este colegio, a a } \\
\text { chiquillos de una tasa de vulnerabilidad altísima [...]". (4,30) }\end{array}$ \\
\hline
\end{tabular}

\section{DISCUSIÓN Y CONCLUSIONES}

Los resultados presentados indican que para los directores el compromiso docente es un atributo profesional que se encuentra íntimamente ligado a la noción de "ser profesor", como una forma de valoración del rol que se desempeña y como una disposición hacia un algo (los estudiantes, la escuela) que se expresa como una entrega extraordinaria más allá de lo que señalan los límites del contrato formal. El compromiso docente involucra una motivación personal por mejorar marcando una diferencia en el quehacer, y por lo tanto, sería un rasgo selectivo que distingue a unos docentes de otros. En este sentido, conecta con dimensiones tales como valores, pasión y creencias; elementos que invitan a comprenderlo como parte de aquellos aspectos que van configurando la identidad docente en conjunto con una mayor conciencia del rol profesional. En este sentido, si bien en las creencias de los directores se habla del compromiso hacia la escuela o hacia los estudiantes, esto puede entenderse también como parte de las dimensiones en que el compromiso se expresa y 
que están íntimamente ligadas al contexto en el que les toca enseñar. Así también, según Bolívar (2010), lo plantean algunos investigadores al definir el compromiso como un estado voluntario en que la motivación intrínseca hacia los objetivos y valores de una institución (escuela) inspira esfuerzos más allá de las expectativas mínimas. Se corresponde con los relatos de los entrevistados cuando aluden a un compromiso como "entrega", como una forma de darse con los alumnos, estar dispuesto a todo, más allá de lo que está establecido por un contrato con el empleador. Esto implica que el compromiso no se dirige a la escuela como objeto, sino a lo que la escuela representa en la experiencia profesional de cada profesor.

En las investigaciones previas (Sun, 2014; Hussen, Awgichew \& Thesome, 2016), se identificaron cuatro grandes dimensiones del compromiso docente: compromiso con la enseñanza, compromiso con los estudiantes, compromiso con la escuela y compromiso con el cambio. De ellas, al menos tres dimensiones se reconocen en los elementos que, desde el relato de los participantes se categorizaron como manifestaciones del compromiso docente: una relación cercana y cálida con los estudiantes, el cumplimiento del rol formal como profesor, la preocupación por el aprendizaje, el manejo de la disciplina de aula, el rol de profesor jefe y la adecuación a las condiciones de trabajo. Esto puede indicar que desde las creencias de los directores el compromiso docente se manifiesta de un modo que caracteriza el quehacer docente, y que se presenta como un sello actitudinal y comportamental que se aprende en la interacción con otros en un determinado contexto, como se ha planteado desde la investigación haciendo referencia a su carácter situado (Crosswell \& Elliot, 2004).

En relación a los elementos que contextualizan el compromiso docente, adquieren especial relevancia las creencias que los entrevistados develan acerca de la docencia. En ellas se reúnen aspectos principalmente valorativos que sirven como punto de partida para juzgar a los docentes y su trabajo, pero que además reflejan los puntos de quiebre que se han descrito en la literatura respecto de las reconfiguraciones de la identidad docente. Así por ejemplo, cuando los entrevistados señalan que la docencia se define por su rol social al tiempo que la reconocen como un trabajo de poco impacto y que se ha perdido el respeto y orgullo profesional, se replica lo que Bolívar, Gallego, León y Pérez (2005) describen en cuanto a la imagen desdoblada que experimentan los docentes, en la que se conjuga el discurso heredado de la dimensión vocacional y trascendente de la tarea de enseñar, con la deslegitimación y pérdida del prestigio social.

Al tener en cuenta las creencias sobre la docencia en su relación con las creencias sobre el compromiso docente, se corrobora de algún modo el vínculo que en la literatura se ha descrito como una relación dinámica entre compromiso e identidad docente. Los resultados indican que las creencias sobre el compromiso docente se construyen, en el relato de los sujetos, sobre creencias más amplias relativas a la docencia, tal vez actuando estas últimas como un soporte. Esto se corresponde con los antecedentes presentados previamente y que apuntan a la configuración que adquiere el compromiso docente en la construcción que los profesores hacen de su identidad. No se trata de una especie de subconjunto de la identidad docente, más bien, el compromiso docente sería un elemento que articula los aspectos identitarios expresados en el ejercicio de la docencia, de cara a las demandas del entorno. El compromiso estaría expresando un atributo que define al profesor en su rol y en relación con su trabajo, aportándole una valoración global, no solo en un sentido profesional, sino en un sentido histórico y personal (Day, 2011). Estos mismos elementos, como la valoración del rol, como manifestación de una vocación, y la dimensión histórica de esta construcción, son los que se recogen en las creencias que los sujetos expresan sobre la docencia. 
A modo de conclusión, se propone un modo de entender los resultados aquí descritos como un sistema de creencias, en el que las categorías del Eje A: "Creencias sobre el compromiso docente", pueden ser elementos nucleares, y las categorías del Eje B: "Elementos que contextualizan el compromiso docente", pueden formar parte de dichas creencias pero no las definen, aunque aportan elementos para entender cómo el fenómeno se expresa y se va calibrando en los contextos en que los sujetos construyen, modifican o reafirman sus creencias. En este sentido, las creencias sobre el compromiso docente no serían unidades cerradas sino que estarían conectadas con subgrupos de creencias sobre otros elementos vinculados al compromiso y a la docencia. A partir de esto, se justifica el desarrollar estudios similares en otros grupos de directores y/o docentes más allá del contexto municipal, y observar si los elementos de las creencias sobre el compromiso docente permanecen estables o se incorporan otros aspectos aportados por el entorno.

Los resultados muestran creencias, acerca de los docentes, marcadas por un fuerte acento negativo, lo que les falta, lo que no hacen bien, lo que los aqueja; contrastando con las creencias que ofrecen una visión idealizada respecto del compromiso docente. Cabe cuestionarse si estas creencias sobre el compromiso docente representan una tensión que instala una expectativa acerca de los docentes que es difícil de cumplir, teniendo a la vista lo que en la práctica se cree de ellos. Resulta necesario profundizar en este tema, en particular explorar en la influencia de dichas creencias en el pensamiento de los directores y consecuentemente en sus prácticas de liderazgo, específicamente en aquellos modos de organizar sus escuelas que serán favorecedoras o no del compromiso de sus profesores, y en aquellas iniciativas promotoras del autocuidado en el trabajo docente.

Asimismo, y desde una óptica cualitativa, investigaciones futuras podrían indagar en la relación entre las creencias en sobre el compromiso docente y las prácticas de dirección. Una alternativa plausible, para una indagación en profundidad, podrían ser los estudios de corte etnográfico que, permiten, como señala Restrepo (2018), "la articulación entre las prácticas y los significados de esas prácticas.” (p. 25).

Es importante además abordar en futuras investigaciones focalizadas en el pensamiento de los directores, el modo en que estas creencias sobre el compromiso docente operan frente a las nuevas demandas que las políticas de reformas educativas han venido impulsando y que promueven un liderazgo eminentemente técnico pedagógico (Weinstein, Marfán \& Muñoz, 2012). A modo de ejemplo, podría estudiarse la relación entre con los estándares indicativos de desempeño y el Marco para la Buena Enseñanza.

Un aspecto adicional que este estudio deja abierto es la pertinencia de indagar también sobre las prácticas de liderazgo de los directores y su correspondencia o relación con las creencias aquí descritas. Si bien existe suficiente evidencia que avala que las prácticas de liderazgo tienen influencia en el compromiso de los docentes, es interesante develar en qué medida esta influencia aparece como un propósito explícito en las acciones y decisiones de los líderes escolares, y cómo se relacionan dichos propósitos con sus creencias de base. De este modo, esta investigación, en sus resultados y en las incógnitas que permite enunciar, respalda la necesidad de contar con más información sobre cómo los directores construyen sus creencias, considerando la relación entre éstas y sus decisiones y prácticas. ${ }^{2}$

Las autoras agradecen a quienes evaluaron el manuscrito por sus sugerencias, algunas de las cuales fueron incorporadas en su versión final. 
Estudios Pedagógicos XLVII N 1: 71-90, 2021

CREENCIAS DE DIRECTORES DE ESTABLECIMIENTOS EDUCACIONALES MUNICIPALES ACERCA DEL COMPROMISO DOCENTE

\section{REFERENCIAS BIBLIOGRÁFICAS}

Aguilar, J. (2003). Aproximación a las creencias del profesorado sobre el papel de la educación formal, la escuela y el trabajo docente. Región y Sociedad, 15(26), 73-102. Recuperado el 4 de marzo de 2018 desde http://www.scielo.org.mx/scielo.php?script=sci_arttext\&pid=S1870-39252003000100003

Altun, M. (2017). The Effects of Teacher Commitment on Student Achievement. International Journal of Social Sciences and Educational Studies, 3(3), 51-54. doi:10.23918/ijsses.v3i3p51

Arnett, K. \& Mady, C. (2018). The influence of classroom experience on teacher belief systems: new french second language teachers' beliefs about program options for English language learners and students with learning difficulties. Revue des Sciences de l'Éducation de McGILL 53(3), 590-611. Recuperado el 1 de diciembre de 2019 desde https://www.erudit.org/en/journals/mje/2018-v53-n3mje04479/1058418ar.pdf

Berkovich, I. \& Eyal, O. (2017). Emotional reframing as a mediator of the relationships between transformational school leadership and teachers' motivation and commitment. Journal of Educational Administration, 55(5), 450-468. DOI 10.1108/JEA-07-2016-0072

Bolívar, A., Gallego, M., León, M. \& Pérez, P. (2005). Políticas educativas de reforma e identidades profesionales: El caso de la Educación Secundaria en España. Archivos Analíticos de Políticas Educativas, 13(45), 1-51. Recuperado el 1 de octubre de 2015 desde https://www.redalyc.org/ pdf/2750/275020513045.pdf

Bolívar, A. (2009). Una dirección para el aprendizaje. Revista Iberoamericana sobre Calidad, Eficacia y Cambio en Educación, 7(1), 1-4. Recuperado el 2 de Julio de 2016 desde https://www.redalyc.org/ pdf/551/55170101.pdf

(2010). La lógica del compromiso del profesorado y la responsabilidad del centro escolar. Una revisión actual. Revista Iberoamericana sobre Calidad, Eficacia y Cambio en Educación, $8(2)$, 10-33. Recuperado el 13 de noviembre de 2018 desde https://www.redalyc.org/ pdf/551/55114080002.pdf

(2015). Construir localmente la capacidad de mejora: Liderazgo pedagógico y Comunidad Profesional. In Ponencia presentada en el XIII Congreso Nacional de Investigación Educativa, Chihuahua, México. Recuperado el 02 de septiembre de 2019 de https://www.researchgate.net/ profile/Antonio_Bolivar/publication/285895047_Construir_localmente_la_capacidad_de_mejora_ Liderazgo_pedagogico_y_Comunidad_Profesional/links/566424a808ae418a786d364d.pdf

Cansoy, R. \& Polatcan, M. (2019). The Relationship Between School Principals' Leadership and Teachers' Organisational Commitment: A Systematic Review. Bartın University Journal of Faculty of Education, 8(1), 1-31. Doi: 10.14686/buefad.441189

Cherkowski, S. (2012). Teacher Commitment in Sustainable Learning Communities: A New" Ancient" Story of Educational Leadership. Canadian Journal of Education, 35(1), 56-68. Recuperado el 16 de enero de 2016 desde http://journals.sfu.ca/cje/index.php/cje-rce/article/view/717/1152

Cornejo, M. \& Salas, N. (2011). Rigor y calidad metodológicos: un reto a la investigación social cualitativa. Psicoperspectivas, 10(2), 12-34. Doi: 10.5027/PSICOPERSPECTIVAS-VOL10ISSUE2-FULLTEXT-144

Crosswell, L. (2006). Understanding teacher commitment in times of change. Queensland University of Tecnology. Tesis doctoral. Consultado [21/12/2015] en: http://eprints.qut.edu.au/16238/1/Leanne_ Crosswell_Thesis.pdf

Crosswell, L. \& Elliott, R. (2004). Committed teachers, passionate teachers: The dimension of passion associated with teacher commitment and engagement. Recuperado el 29 de noviembre de 2015 desde http://eprints.qut.edu.au/968/1/cro04237.pdf

Day, C. (2011). Pasión por enseñar. La identidad personal y profesional del docente y sus valores. Madrid, España: Narcea Ediciones.

Dewey, J. (1989). Cómo pensamos: nueva exposición de la relación entre pensamiento y proceso educativo. Series: Cognición y desarrollo humano, 18. Barcelona: Paidós. 
Díaz, C., Martínez, P., Roa, I. \& Sanhueza, M. G. (2010). Los docentes en la sociedad actual: sus creencias y cogniciones pedagógicas respecto al proceso didáctico. Polis, Revista de la Universidad Bolivariana, 3(2), 421-436. Recuperado el 4 de marzo de 2018 de https://scielo.conicyt.cl/scielo. php?script=sci_arttext\&pid=S0718-65682010000100025

Diego-Mantecón, J., Blanco, T., Chamoso, J. \& Cáceres, M., (2019). An attempt to identify the issues underlying the lack of consistent conceptualizations in the field of student mathematics-related beliefs. PLOS ONE 14(11): e224696. Recuperado el 1 de diciembre de 2019 desde https://www. ncbi.nlm.nih.gov/pmc/articles/PMC6834264/pdf/pone.0224696.pdf

Doğan, H. \& Çelik, K. (2019). The Relation between School Administrators' Use of Power Styles and Teachers' Organizational Commitment and Their Job Performance. Egitim ve Bilim, 44(198), 3755. DOI: $10.15390 / \mathrm{EB} .2019 .7943$

Fickel, L. (1999). Democracy is Messy: Exploring the beliefs and Personal Theories of a High School Social Studies Teacher. American Educational Research Association, 1-36. Recuperado el 07 de noviembre de 2018 de https://files.eric.ed.gov/fulltext/ED433282.pdf

Firestone, W. \& Pennel, J. (1993). Teacher Commitment, Working Conditions, and Differential Incentive Policies. Review of Educational Research 63(4), 489-525 DOI: 10.3102/00346543063004489

Flores, R. (2009). Observando Observadores: Una introducción a las Técnicas Cualitativas de Investigación Social. Santiago, Chile: Ediciones Universidad Católica de Chile.

Fuentealba, R. \& Imbarack , P. (2014). Compromiso docente, una interpelación al sentido de la profesionalidad en tiempos de cambio. Estudios pedagógicos (Valdivia), 40(ESPECIAL), 257-273. http://dx.doi.org/10.4067/S0718-07052014000200015

García, M. R. \& Sebastián, C. (2011). Creencias epistemológicas de estudiantes de pedagogía en educación parvularia, básica y media: ¿Diferencias en la formación inicial docente? Psykhe, 20(1), 29-43. Recuperado el 6 de febrero de 2018 desde https://scielo.conicyt.cl/scielo.php?script=sci_art text\&pid=S0718-22282011000100003

Garay, S. \& Uribe, M. (2006). Dirección escolar como factor de eficacia y cambio. Situación de la dirección escolar en Chile. Revista Electrónica Iberoamericana sobre Calidad, Eficacia y Cambio en Educación, 4(4e), 39-64. Recuperado el 22 de mayo de 2016 desde https://www.redalyc.org/ pdf/551/55170101.pdf

Guba, E. (1989). Criterios de credibilidad en la investigación naturalista. En J. Gimeno Sacristán \& A. Pérez Gómez (Eds.), La enseñanza: su teoría y su práctica. (pp. 148-165). Madrid, España: Akal.

Horn, A. \& Murillo, F. (2016). Incidencia de la dirección escolar sobre el compromiso de los docentes: Un estudio multinivel. Psicoperspectivas, 15(2), 64-77. Doi: 10.5027PSICOPERSPECTIVASVOL15-ISSUE2-FULLTEXT-746

Hutner, T. \& Markman, A. (2016). Proposing an operational definition of science teacher beliefs. Journal of Science Teacher Education, 27(6), 675-691. Doi: 10.1007/s10972-016-9480-5

Hussen, A., Awgichew, S. \& Thesome, T. (2016). Teachers Professional Commitment towards Students Learning, their Profession and the Community in Eastern Ethiopian Secondary Schools. Journal of Teacher Education and Educators, 5(3), 289-314. Recuperado el 29 de Julio de 2019 desde http:// jtee.org/document/issue11/MAKALE3.pdf

Jiménez, A. \& Feliciano, L. (2006). Pensar el pensamiento del profesorado. Revista española de pedagogía, (233), 105-122. Recuperado el 3 de marzo de 2018 desde https://www.jstor.org/stable/23765975

Liu, P. (2015). Motivating teachers' commitment to change through transformational school leadership in Chinese urban upper secondary schools. Journal of Educational Administration, 53(6), 735-754. Doi: 10.1108/JEA-02-2014-0026

Mellado, M. E. \& Chaucono, J. (2015). Creencias pedagógicas del profesorado de una escuela rural en el contexto mapuche. Actualidades Investigativas en Educación, 15(3). https://doi.org/10.15517/ aie.v15i3.20924

Murillo, F., Martínez, C. \& Hernández, R. (2011). Decálogo para una enseñanza eficaz. REICE. Revista Electrónica Iberoamericana sobre Calidad, Eficacia y cambio en Educación, 9(1), 6-27. Recuperado 
Estudios Pedagógicos XLVII N 1: 71-90, 2021

CREENCIAS DE DIRECTORES DE ESTABLECIMIENTOS EDUCACIONALES MUNICIPALES ACERCA DEL COMPROMISO DOCENTE

el 10 de noviembre de 2019 desde http://www.rinace.net/reice/numeros/arts/vol9num1/art1.pdf

Mwaniki, G., Kiumi, J. \& Ngunjiri, M. (2018). Relationship between Teacher Commitment to Students Learning Needs and Level of Students' Discipline in Public Secondary Schools in Naivasha SubCounty, Kenya. International Journal of Scientific Research and Management, 6(04), 274-281. Doi: 10.18535/ijsrm/v6i4.el09

Pajares, F. (1992). Teachers' Beliefs and Educational Research: Cleaning Up a Messy Construct. Review of Educational Research, 62(3), 307-332. https://doi.org/10.3102/00346543062003307

Pino, F. (2019)._Compromiso docente: una comprensión desde las creencias y prácticas de directores de escuelas municipales. (Tesis de Magister). Universidad de Santiago de Chile, Santiago, Chile.

Raczynski, D. (2012). Realidad de la educación municipal en Chile: ¿Liderazgo del sostenedor municipal?. En J. Weinstein \& G. Muñoz. (Eds.), ¿Qué sabemos sobre los directores de escuela en Chile? (pp. 181-217). Santiago, Chile: Fundación Chile y CEPPE-UC.

Reeves, M. (2012). Efectos del liderazgo directivo en escuelas con altos niveles de vulnerabilidad social. In J. Weinstein, \& G. Muñoz (Eds.), ¿Qué sabemos sobre los directores de escuela en Chile? (pp. 307-320). Santiago, Chile: Fundación Chile y CEPPE UC.

Restrepo, (2018). Etnografía. Alcances, técnicas y éticas. Lima: Universidad Nacional Mayor de San Marcos. Recuperado el 19 de agosto de 2020 desde https://www.aacademica.org/eduardo.restrepo/3

Rojas, M. T. (2014). Las creencias docentes: delimitación del concepto y propuesta para la investigación. Revista Electrónica: Diálogos Educativos, 14(27), 89-112. Recuperado el 07 de febrero de 2018 desde http://www.dialogoseducativos.cl/revistas/n27/rojas

Shila, J. \& Sevilla, A. (2015). The impact of the principals' leadership style on teachers' job satisfaction and organizational commitment: An Indian perspective. International Journal of Education \& Management Studies, 5(1), 1-7. Recuperado el 5 de agosto de 2019 desde https://www.questia.com/ library/journal/1P3-3655877501/the-impact-of-the-principals-leadership-style-on

Shirrell, M. (2016). New principals, accountability, and commitment in low-performing schools. Journal of Educational Administration, 54(5), 558-574. Doi: 10.1108/JEA-08-2015-0069

Shoaib, H. \& Khalid, I. (2017). Commitment with the Teaching Profession: Demographical View of Teacher Educators. Pakistan Journal of Education, 34(2), 19-36. Recuperado el 20 de julio de 2019 desde https://pdfs.semanticscholar.org/c6b0/0ef10b6861b71226d6f160e77de2bcfd506a.pdf

Strauss, A. \& Corbin, J. (2002). Bases de la investigación cualitativa. Técnicas y procedimientos para desarrollar la teoría fundamentada. Colombia: Editorial Universidad de Antioquia.

Sun, J. (2014). Conceptualizing the critical path linked by teacher commitment. Journal of Educational Administration, 53(5), 597-624. Doi: 10.1108/JEA-05-2013-0063

Sun, J. \& Leithwood, K. (2015). Leadership effects on student learning mediated by teacher emotions. Societies, 5(3), 566-582. Doi:10.3390/soc5030566

Tapia, C., Becerra, S., Mancilla, J. \& Saavedra, J. (2011). Liderazgo de los directivos docentes en contextos vulnerables. Educación y Educadores, 14(2), 389-409.

Valenzuela, P. \& Horn, A. (2012). Influencia del liderazgo directivo en los resultados de los estudiantes. In J. Weinstein, \& G. Muñoz (Eds.), ¿Qué sabemos sobre los directores de escuela en Chile? (pp. 325-348). Santiago, Chile: Fundación Chile y CEPPE UC.

Vasilachis, I. (2009). La investigación cualitativa. En I. Vasilachis (Coord.), Estrategias de investigación cualitativa (pp. 23-64). Barcelona, España: Gedisa.

Weinstein, J. (2009). Liderazgo directivo, asignatura pendiente de la Reforma Educacional Chilena. Revista Estudios Sociales, 117, 123-148. http://dx.doi.org/10.5027/psicoperspectivas-Vol9-Issue2fulltext-112

Weinstein, J., Marfán, J. \& Muñoz, G. (2012). ¿Colegas o jefes? La visión de los docentes sobre el liderazgo directivo en Chile. In J. Weinstein, \& G. Muñoz (Eds.), ¿Qué sabemos sobre los directores de escuela en Chile? (pp. 255-280). Santiago, Chile: Fundación Chile y CEPPE UC. 